Article

\title{
A Novel Variable Stiffness Mechanism Capable of an Infinite Stiffness Range and Unlimited Decoupled Output Motion
}

\section{Stefan Groothuis *, Raffaella Carloni and Stefano Stramigioli}

Robotics and Mechatronics Group, CTIT Institute, University of Twente, P.O. Box 217, 7500 AE

Enschede, The Netherlands; E-Mails: r.carloni@utwente.nl (R.C.); s.stramigioli@utwente.nl (S.S.)

* Author to whom correspondence should be addressed; E-Mail: s.s.groothuis@utwente.nl;

Tel.: +31-(0)53-489-2707.

Received: 30 December 2013; in revised form: 1 May 2014 / Accepted: 4 May 2014 /

Published: 3 June 2014

\begin{abstract}
In this paper, a novel variable stiffness mechanism is presented, which is capable of achieving an output stiffness with infinite range and an unlimited output motion, i.e., the mechanism output is completely decoupled from the rotor motion, in the zero stiffness configuration. The mechanism makes use of leaf springs, which are engaged at different positions by means of two movable supports, to realize the variable output stiffness. The Euler-Bernoulli leaf spring model is derived and validated through experimental data. By shaping the leaf springs, it is shown that the stiffness characteristic of the mechanism can be changed to fulfill different application requirements. Alternative designs can achieve the same behavior with only one leaf spring and one movable support pin.
\end{abstract}

Keywords: variable stiffness mechanism; hypocycloid gearing; leaf spring; infinite stiffness range; unlimited motion

\section{Introduction}

Traditional robotic systems have stiff structures and are actuated using stiff joints, i.e., they use different types of electric motors, possibly with a gear reduction. This can be desirable for industrial applications to achieve a large position control bandwidth and, therefore, high positioning accuracy and fast operation. However, more and more robotic systems working in cooperation with humans are developed for which a severe demand on safety is placed. This requires a paradigm shift from stiff robots to compliant robots, which can be implemented by, for instance, using compliant manipulator segments 
or compliant joints. Embedding compliant behavior in joints was first done using active impedance control [1], which mimics the behavior of an elastic element through control. A good performance can be achieved using this strategy for sufficiently slow interaction, but since control bandwidth is always limited, the controller cannot react to arbitrary high frequency interaction, e.g., shocks and impacts. Therefore, a physical elastic element has been introduced to realize series elastic actuators (SEA) to ensure intrinsic compliance [2]. By measuring the deflection of the elastic element, the force that is generated in a joint can be calculated, and therefore, robust interaction control is possible. Moreover, the physical elastic element ensures an infinite disturbance bandwidth. A variable stiffness actuator (VSA) [3] is a further development by being able to adjust the perceived actuator stiffness to meet requirements imposed by a changing environment or operating condition. Moreover, the intrinsic system dynamics can be tuned to achieve energy efficiency [4]. This novel actuation concept is necessary for increased safety and seems highly promising in, for instance, the assistive robotics field [5], which relies on human-robot interaction.

Many different implementations of VSA mechanisms have been presented in the literature, which can be roughly classified into three major groups [3], i.e., the spring preload group (of which an antagonistic realization closely resembles a human joint) [6-8], the group in which the transmission between load and spring is changed [9-14] and the group where the physical properties of the spring are changed [15-17]. Each design has its own characteristic properties: the spring preload designs rely on adjusting the preload of elastic elements, which allows them to store a considerable amount of energy, but requires them to put energy in the elastic elements to change the stiffness, which may not be retrievable [18], while their stiffness range is finite; the variable transmission between load and spring designs require no energy to be put in the elastic elements to change the stiffness, but it is more difficult to store a considerable amount of energy. An infinite stiffness range is, however, possible; the designs where the physical properties of the spring are changed also require no energy to be put into the elastic elements to change their stiffness, and a considerable amount of energy can be stored in the elastic elements, although an infinite stiffness range within this class has not yet been shown in literature.

In this paper, the novel design of a variable stiffness mechanism (VSM) is presented, which fits in the group in which the transmission between load and spring is changed. The novelty lies in the property that when the mechanism is in its zero stiffness configuration, the output is completely decoupled from the input (the rotor) and an unlimited output motion is then possible (unconstrained, infinite rotation). Therefore, the output behavior will only be a result of the dynamics of the output load. Moreover, the actuator is capable of achieving an infinite stiffness range (from truly zero stiffness to infinite stiffness, i.e., the parasitic mechanical stiffness), which was not shown before using leaf springs.

This paper is structured as follows: first, the conceptual design is presented in Section 2. Section 3 elaborates on the mechanism model, and Section 4 shows the experiments performed on a setup very similar to the prototype mechanism. The paper is concluded with Section 5, and the possible future research focus is presented in Section 6. 


\section{Conceptual Design}

This section elaborates on the conceptual design, showing the working principle of the VSM and the key design features that enable an infinite stiffness range and completely decoupled and infinite output motion from the input.

\subsection{The Variable Stiffness Mechanism and Its Working Principle}

The novel VSM, shown in Figure 1, consists of a stator, a rotor and an output. In this VSM, the compliance is created between the rotor and the output by using two leaf springs that connect to the output at their ends and connect to the rotor via support pins, fixed on the gears of a hypocycloid gearing mechanism. These pins can move in between the leaf springs in a straight line, imposed by the hypocycloid gearing mechanism, allowing variable compliance in between the rotor and the output. The two planet gears are connected to and rotated by the planet carrier.

Figure 1. The novel variable stiffness mechanism creates compliance between the rotor and output by means of leaf springs, and it is made variable by means of support pins that can be moved in between the leaf springs by using a hypocycloid gearing mechanism.
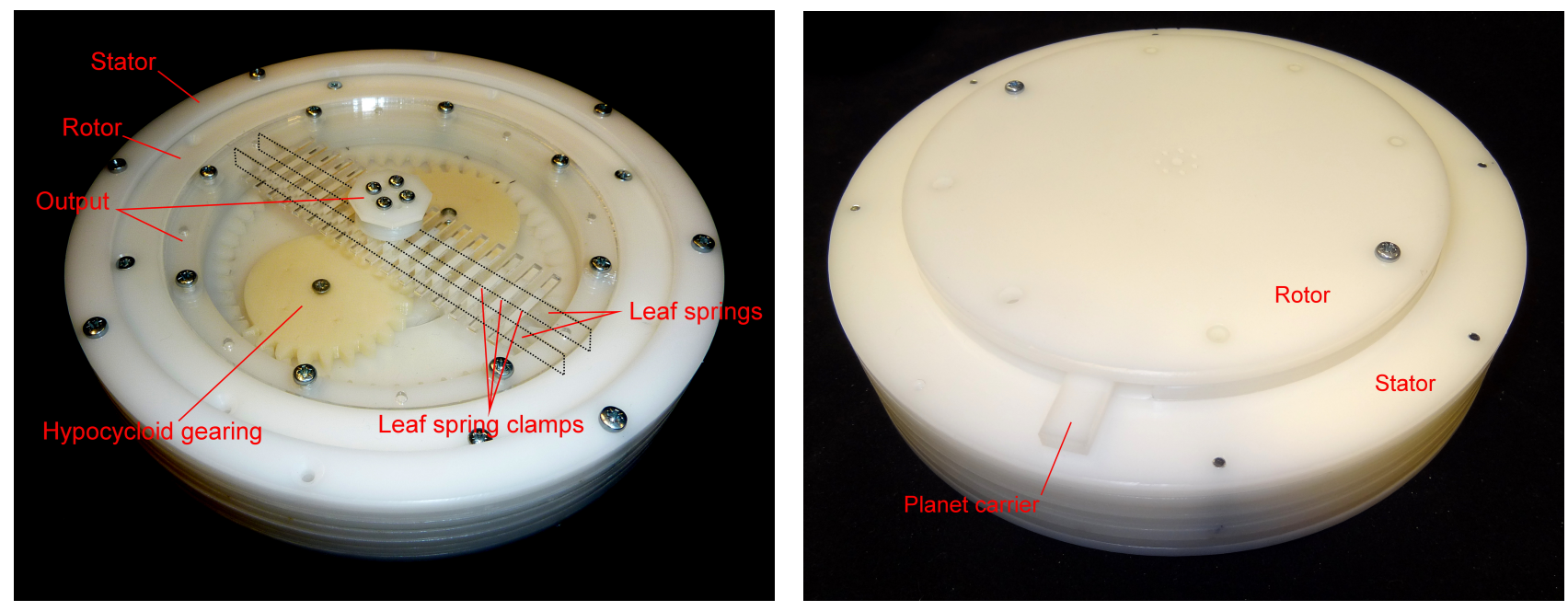

The working principle of the concept (of only one leaf spring) is shown in Figure 2. The principle is based on the group in which the transmission between load and spring is changed. A force couple, $F$, is applied on the undeflected leaf spring (in grey), which causes it to deflect (in black). The location of the movable supports (with coordinate $x_{0}$ ) influences the deflection shape of the entire leaf spring. For an increasing $x_{0}$, the transmission between load and spring is changed, and therefore, the perceived stiffness at the force application points is increasing. When the supports are located at both ends of the leaf spring, an infinite stiffness is felt. When the supports are joined together in the middle of the leaf spring, i.e., $x_{0}=0$, a zero stiffness is felt, and moreover, the leaf spring is free to rotate about its center, due to force couple $F$. In the VSM, this means that the output is completely decoupled from the rotor and that the output only shows dynamics imposed by the load. Note that the active length is always the entire leaf spring, so the entire leaf spring determines the perceived stiffness. 
Note that the device presented here is not yet an actuator in the sense that it has motors and can deliver power to a load. Currently, it is a conceptual mechanism to which actuators can be connected.

Figure 2. The working principle of the variable stiffness mechanism. The deflection shape of the leaf spring is varied by changing the location of the supports, and therefore, the perceived stiffness at the location of the force application points is changed.

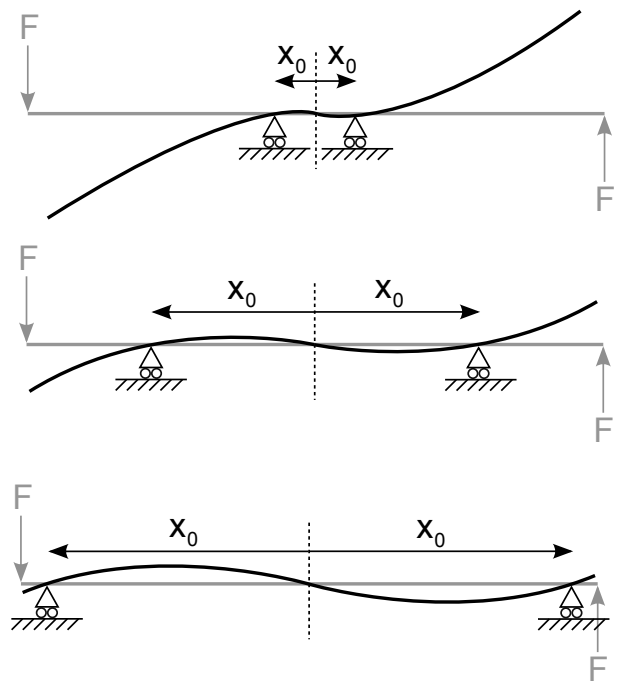

\subsection{Support Pins}

The support pins, at location $x_{0}$, have a special shape (the designed shape (note that other pin shapes similar to this are possible, such as a helical shape) is shown in Figure 3), such that they can join together in the center of the VSM to form a perfect cylinder, such that the output is decoupled from the input. This means that the output can rotate infinitely, independent of the input. Note that when the output is rotated exactly perpendicular to the imaginary line along which the supports move, a singularity occurs, because of the leaf springs constraining the supports to moving apart. Since the rotor movement is also independent of the input, a controller should keep track of the output movement during free rotation and adjust the rotor position accordingly to prevent this singularity from occurring. When moving apart, the shape of the support pins ensures that the space between the two leaf springs is completely filled, leaving no room for play, at any location along the leaf springs, which is made explicit in Figure 4.

\subsection{Hypocycloid Gearing Mechanism}

The supports pins should be moved in a straight line along the leaf spring, which is achieved using a hypocycloid gearing mechanism (similar to a planetary gearing system, previously proposed in [14]) and shown in Figure 5. It consists of one ring gear and two planet gears, and the supports are fixed to the planet gears. The ring gear is fixed to the rotor, and the planet gears are fixed to and rotated by the planet carrier. Because the pitch diameter of the planet gears is half the pitch diameter of the ring gear, a point on the planet gear pitch diameter tracks a straight line. Note that half of the planet gear teeth should be removed to allow the two planets to rotate. In the proposed VSA mechanism, there are two leaf springs placed at a small distance from each other in between which the supports can move. To ensure the leaf 
springs are held together at the same distance and deflect as if they are one leaf spring, they are held together by small clamps.

Figure 3. The pin used as one of the two supports for the leaf spring.
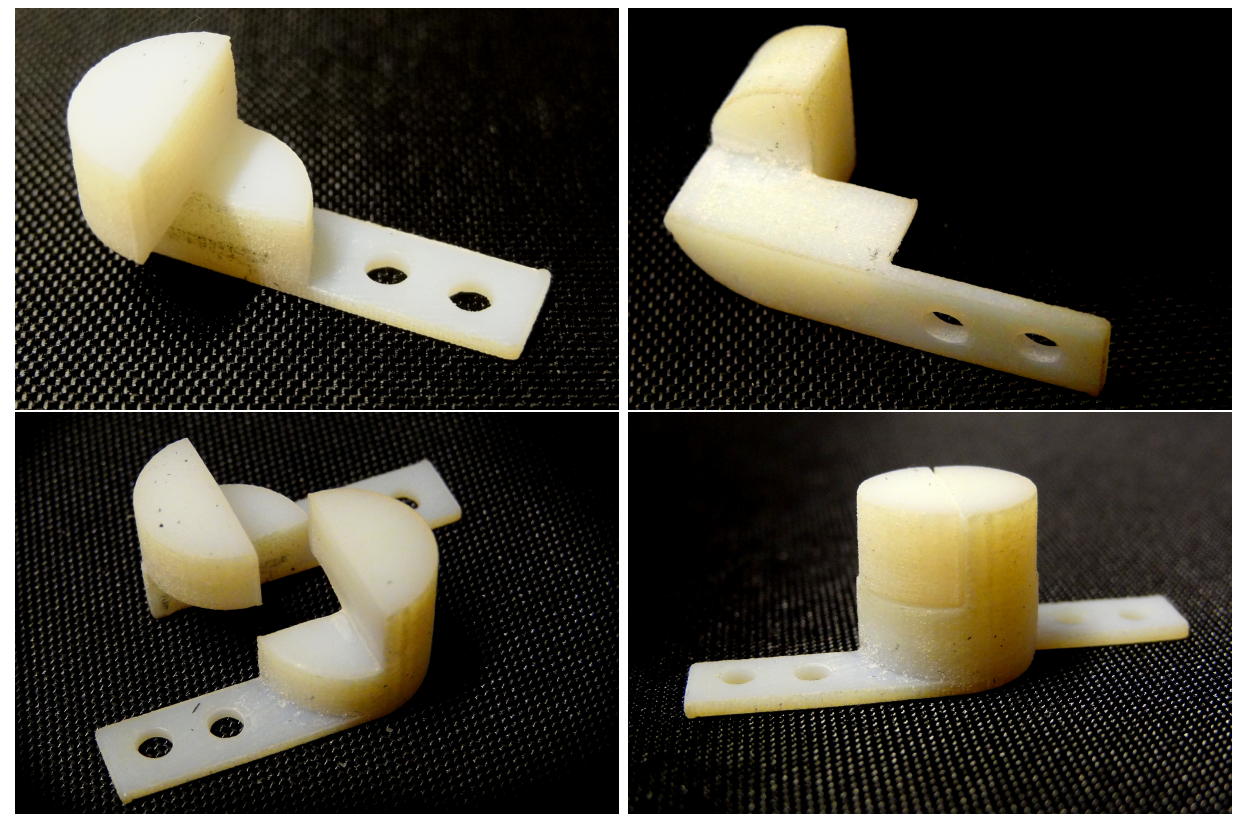

Figure 4. Schematic representation of the motion of the supports in between the leaf springs. Contact with both leaf springs is always ensured, because of the pin shape (shown in red).

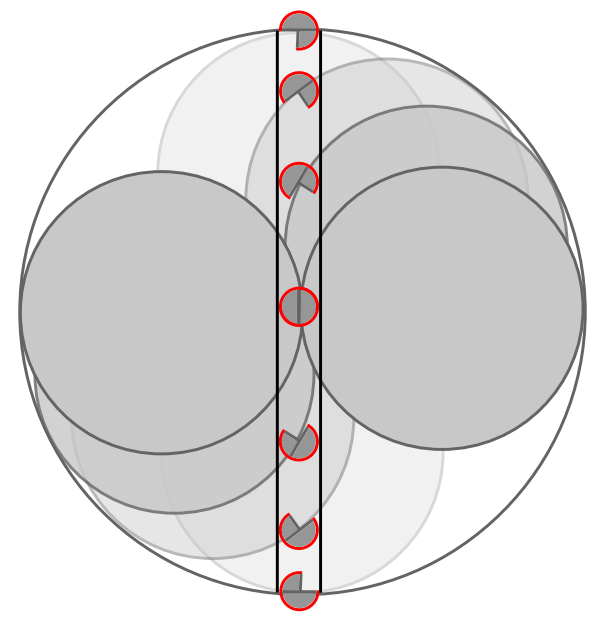

\section{Modeling}

This section elaborates on the VSM modeling. A leaf spring was modeled as a beam to analyze its deflection for specific support locations and applied forces. Although the mechanism consists of two leaf springs, only one leaf spring is modeled, since an otherwise highly complex model would likely result from the modeling of the coupling between the leaf springs by the clamps. A finite element model (FEM) of the support pin was used to analyze the stress on the pins as a result of the actuator torque. 
Figure 5. The hypocycloid gearing mechanism used to move the supports in a straight line along the leaf spring.

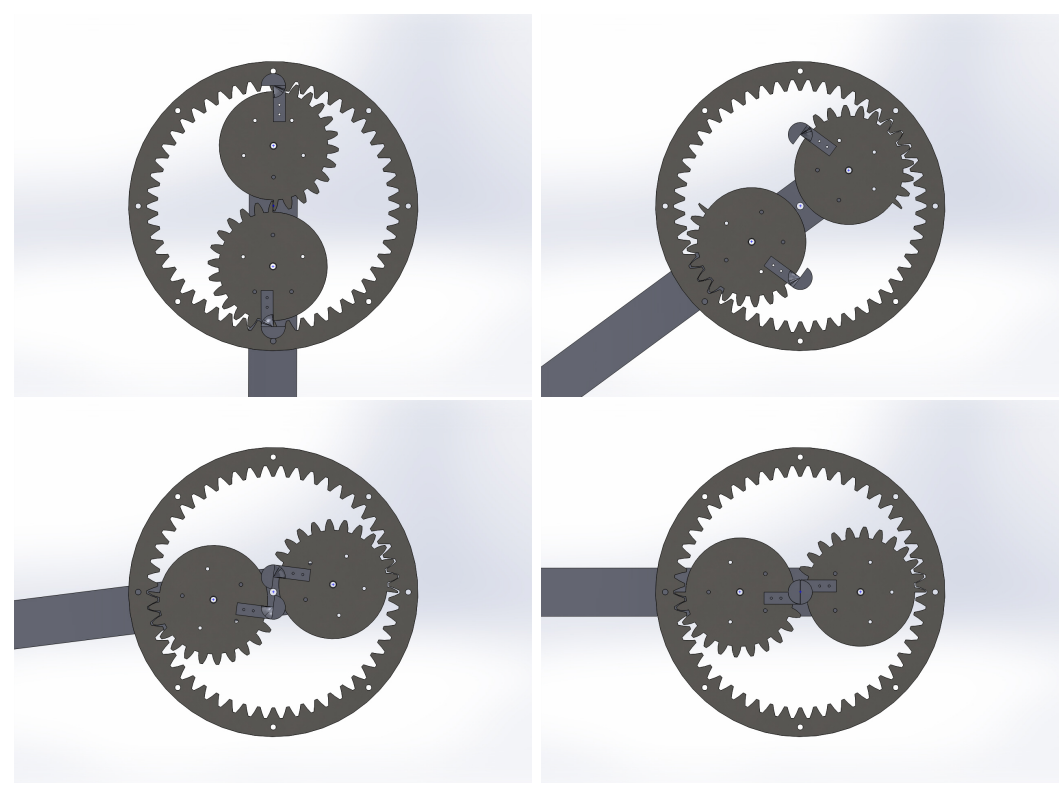

\subsection{Leaf Spring}

The leaf spring is modeled as a doubly-supported, doubly-overhanging rectangular Euler-Bernoulli beam with a force couple on the ends due to the mechanism output torque (refer to Figure 2). A Euler-Bernoulli model of this beam is justified versus the use of, for example, the Timoshenko beam model, taking shear deformation into account, since the leaf spring is relatively thin compared to its other dimensions. Hence, assuming that a cross-section is always perpendicular to the neutral axis is justified.

The geometry of the beam and the coordinate frame that is used, which is placed in the center of the beam, is shown in Figure 6 . The beam deflection in the $z$-direction is a function of the $x$-coordinate.

Figure 6. Geometry and coordinate usage of a generic beam.

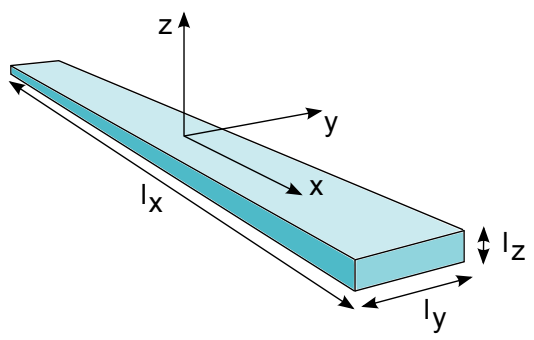

The general linear Euler-Bernoulli beam equation is given by:

$$
\frac{\mathrm{d}^{2}}{\mathrm{~d} x^{2}}\left(B(x) \frac{\mathrm{d}^{2}}{\mathrm{~d} x^{2}}(v(x))\right)=q(x)
$$

where the term $B(x):=E I_{x x}(x)$ is a function of Young's modulus $E$ (It is assumed that the leaf spring has uniform density, i.e., Young's modulus $E$ is constant over the material), the area moment of inertia, $I_{x x}, v(x)$, is the beam deflection at coordinate $x$, and $q(x)$ is the loading profile on the beam. 
Expanding the terms, this equation can be given by:

$$
\frac{\mathrm{d}^{2}}{\mathrm{~d} x^{2}} E I_{x x}(x) \frac{\mathrm{d}^{2}}{\mathrm{~d} x^{2}} v(x)+2 \frac{\mathrm{d}}{\mathrm{d} x} E I_{x x}(x) \frac{\mathrm{d}^{3}}{\mathrm{~d} x^{3}} v(x)+E I_{x x}(x) \frac{\mathrm{d}^{4}}{\mathrm{~d} x^{4}} v(x)=q(x)
$$

This is a fourth order, first degree, ordinary differential equation with variable coefficients.

For a uniform beam, i.e., a beam with constant geometry $E I_{x x}(x) \rightarrow E I_{x x}$, the first two terms are zero, and the equation becomes:

$$
E I_{x x} \frac{\mathrm{d}^{4}}{\mathrm{~d} x^{4}} v(x)=q(x)
$$

This is a fourth order, first degree, ordinary differential equation with constant coefficients.

The beam deflection can be solved by separately taking into account the individual loading segments and choosing proper boundary conditions to form a continuous solution. However, the loading profile can also be specified in a way such that it is valid over the complete beam length and solving it for the complete beam at once. The loading on the leaf spring consists of four forces, as shown in Figure 7.

Figure 7. Force profile on the leaf spring, consisting of four forces: two applied forces, $F$, and two reaction forces on the support pins, $R_{a}$ and $R_{b}$.

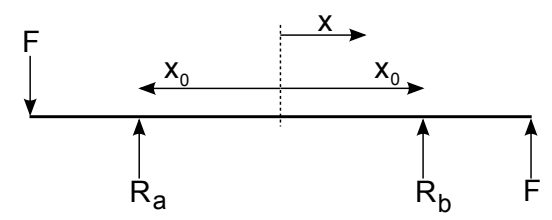

The loading $q(x)$ can be given as:

$$
q(x):=-F \cdot \delta\left(x+\frac{1}{2} l_{x}\right)+R_{a} \cdot \delta\left(x+x_{0}\right)+R_{b} \cdot \delta\left(x-x_{0}\right)+F \cdot \delta\left(x-\frac{1}{2} l_{x}\right)
$$

where $F$ is the force on the ends of the leaf spring due to the output torque, $R_{a}$ and $R_{b}=-R_{a}$ are the reaction forces at the supports and $\delta(x-\alpha)$ is the Dirac pulse located at $x=\alpha$.

The fourth order differential equation of (1) needs four boundary conditions to solve for the deflection, $v(x)$. Two boundary conditions are given at the support locations, since there, the deflection is zero. Figure 8 gives the other two boundary conditions, namely the slope at the ends of the leaf springs should be coincident with the center of the mechanism. The boundary condition equations are given by:

$$
\begin{aligned}
& v\left(x_{0}\right)=0 \\
& v\left(-x_{0}\right)=0 \\
&\left.\frac{\mathrm{d}}{\mathrm{d} x} v(x)\right|_{x=\frac{1}{2} l_{x}}:=\phi\left(\frac{1}{2} l_{x}\right)=\frac{v\left(\frac{1}{2} l_{x}\right)}{\frac{1}{2} l_{x}} \\
&\left.\frac{\mathrm{d}}{\mathrm{d} x} v(x)\right|_{x=-\frac{1}{2} l_{x}}:=\phi\left(-\frac{1}{2} l_{x}\right)=\frac{v\left(\frac{1}{2} l_{x}\right)}{\frac{1}{2} l_{x}}
\end{aligned}
$$

The leaf spring bends in the $z$-direction, so the area moment of inertia, $I_{x x}$, for a uniform beam is given by:

$$
I_{x x}:=\frac{l_{y} l_{z}^{3}}{12}
$$


Young's modulus $E$ depends on the material that is used; in this mechanism, PETGcopolyester with a Young's modulus of $1.9 \mathrm{GPa}$ was used.

Figure 8. The leaf spring (thick solid line) is fixed to the output of the variable stiffness actuator (VSA) mechanism (grey ring), which causes the leaf spring slope at its ends (dashed line) to be coincident with the mechanism center and perpendicular to the tangent of the output ring.

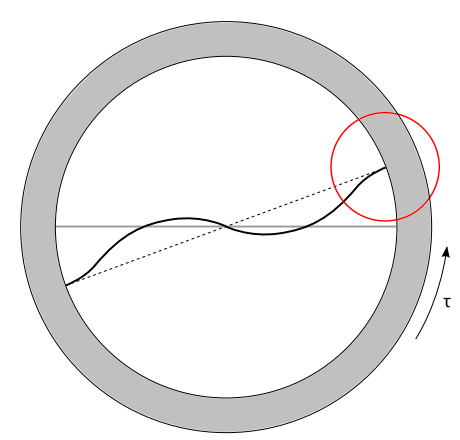

When solving the equation in (1) together with the boundary conditions of (2) for a uniform beam, with lengths $l_{x}=100 \mathrm{~mm}, l_{y}=8 \mathrm{~mm}$ and $l_{z}=1 \mathrm{~mm}$, the beam shape for different settings of the support location with coordinate $x_{0}$ is shown in Figure 9. Indeed, for increasing $x_{0}$, the beam deflection decreases at a constant force, $F$, and therefore, the perceived stiffness increases. Note the particular beam shape at the ends, caused by clamping the leaf spring in the output.

Figure 9. The beam shape for several settings of the support location, $x_{0}$, under a constant applied force, $F$. Note the particular beam shape near the ends, caused by clamping the leaf spring in the mechanism output.

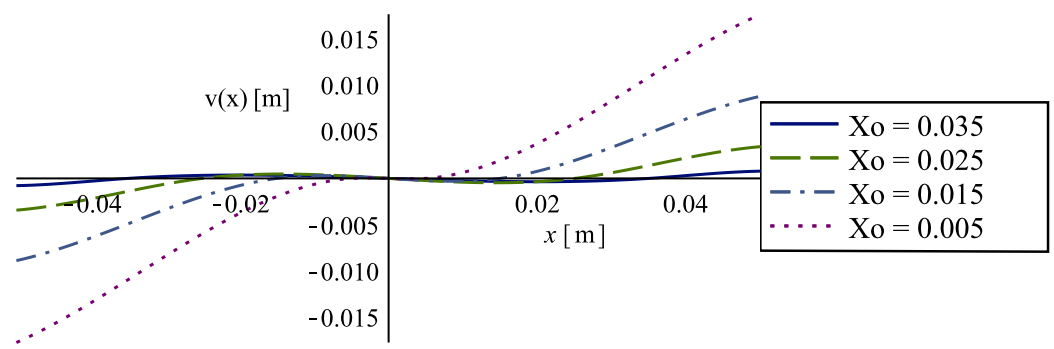

When the leaf spring is not uniform, but either variable over length $l_{y}$ or $l_{z}$ as a function of coordinate $x, I_{x x}$ becomes a function of $x$. This affects the stiffness of the beam, which is a desirable feature of the proposed mechanism, since a different stiffness characteristic can be very easily achieved by only adjusting the shape of the leaf spring. When assuming that only length $l_{y}$ is variable over coordinate $x$, which is realized more easily than a variable, $l_{z}$, the area moment of inertia becomes:

$$
I_{x x}(x):=\frac{l_{y}(x) l_{z}^{3}}{12}
$$

In Section 4, three different leaf spring shapes are measured to make the shaping of the stiffness characteristic explicit. Equations (1) and (2) of these non-uniform beams were solved numerically, and those results are plotted together with the measurements in Section 4. 
Note that the model presented here is based on linear beam theory, and therefore, the model is less accurate for larger deflections.

\subsection{Support Pins}

The support pins should have a special shape to allow two of those pins to join together, while still ensuring that the complete space between two leaf springs in the mechanism is filled, such that there is no play between the output and the rotor. The support pins should also be able to withstand the forces as a result of force $F$ on the leaf spring.

The force on a support pin is equal to $F$ when a support is located at $x_{0}=\frac{1}{2} l_{x}$ and increases when $x_{0}$ decreases. The maximum support force occurs for $0<x_{0} \ll \epsilon$, with $\epsilon$ infinitely small, i.e., at a location infinitely close to zero, but not equal to zero. For a constant output force, $F$, this would result in an impossibly large deflection of the leaf spring. Therefore, to calculate the maximum support force, its deflection needs to be limited. Figure 10 shows the force in one of the supports, for a constant applied maximum force $F=F_{\text {max }}$ and for an applied force, $F$, that causes a certain constant maximum leaf spring deflection $F=F_{v_{\max }}$. For a maximum force $F_{\max }=200 \mathrm{~N}$ (corresponding to an arbitrary maximum output torque of $\tau=10 \mathrm{Nm}$ ) and a maximum allowable leaf spring deflection $v_{\max }=\frac{1}{4} l_{x}$, the maximum support force is calculated to be $R_{a \max }=238.4 \mathrm{~N}$ at $x_{0}=42.0 \mathrm{~mm}$.

Figure 10. Support force $R_{a}$ as a function of support location $x_{0}$. Two plots are shown: $F=F_{\max }$ shows the support force if always a force $F=F_{\max }$ is allowed to be applied on the leaf spring, and $F=F_{v_{\max }}$ shows the support force when a certain force, $F$, is applied with which a certain maximum deflection $v(x)=v_{\max }$ is achieved. Their crossing is the maximum possible support force, $R_{a \max }$.

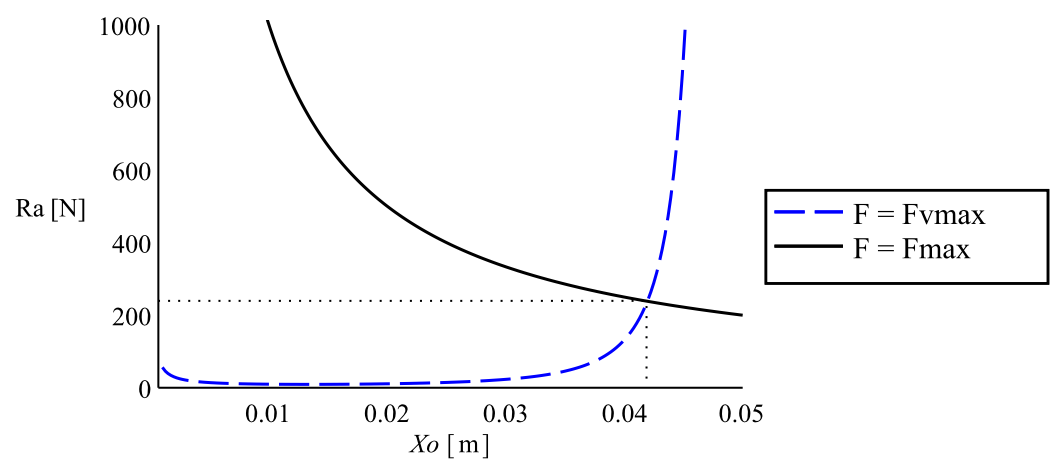

The maximum force, $F_{\max }$, on the support pin occurs at a certain support location, $x_{0}$, and, therefore, under a certain angle defined by the motion of the hypocycloid gearing mechanism. It is calculated that this angle for $x_{0}=42.0 \mathrm{~mm}$ is $\theta_{\max }=32.96^{\circ}$. That force is applied on a finite element model of the support pin (refer to Figure 11) to obtain the stress distribution inside the pin. Using regular alloy steel with a Young's modulus of $210 \mathrm{GPa}$ and a yield strength of $620 \cdot 10^{6} \mathrm{~N} / \mathrm{m}^{2}$, the safety factor using this particular shape is 3.10 . 
Figure 11. The stress analysis of the support pin fixed to a gear of the hypocycloid gearing mechanism. The applied loading is given by the maximum force on the pin under a certain angle.

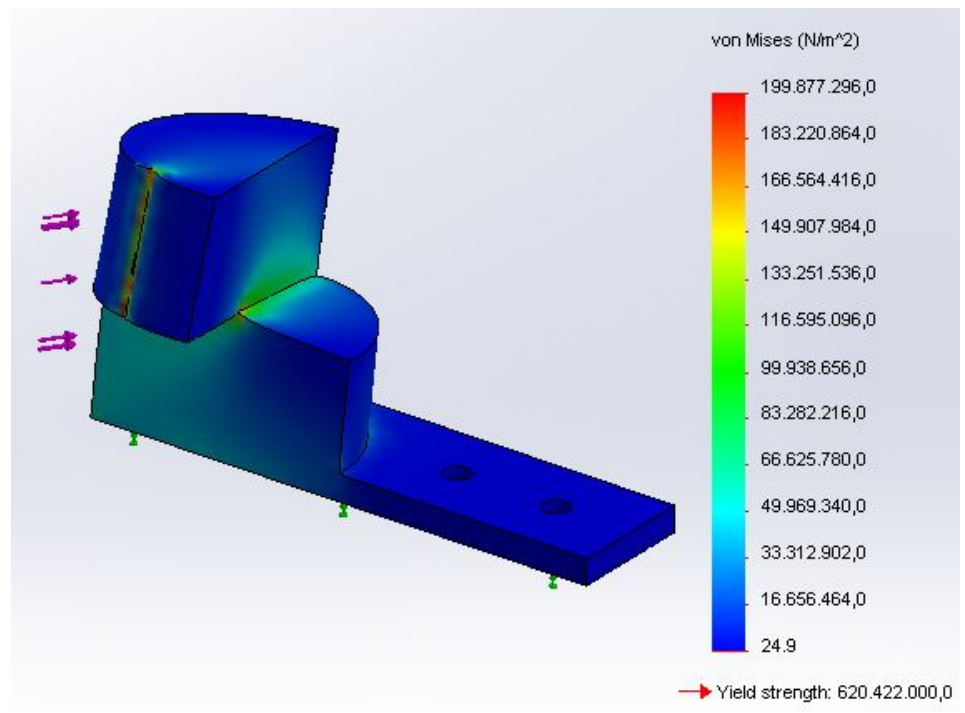

\subsection{Hypocycloid Gearing Mechanism}

Coordinate $x_{0}$ is a function of the planet carrier angle in the hypocycloid gearing mechanism (the angle that the bar to which the two planet gears are fixed makes with respect to the ring gear; see Figure 5), $\theta_{p c}$, and the pitch diameter of the planet gear, $D_{p}$, and is given by $x_{0}=D_{p} \sin \left(\theta_{p c}\right)$. Here, it is assumed that the bottom right figure of Figure 5 represents a planet carrier angle of $\theta_{p c}=0 \mathrm{rad}$, and the top left figure represents an angle of $\theta_{p c}=\frac{1}{2} \pi \mathrm{rad} . D_{p}$ is $50 \mathrm{~mm}$ in this setup. Readers are referred to [14] for the full kinematic analysis.

\section{Experiments}

Experiments have been performed to measure the stiffness characteristic of the proposed variable stiffness mechanism. As stated before, the stiffness characteristic that is a function of the support location, $x_{0}$, can be changed by shaping the leaf springs. Three different PETG leaf spring shapes were used during the measurements, namely a uniform shape, a negative parabola cutout and a positive parabola cutout, as is shown in Figure 12. In 1, the height is $8 \mathrm{~mm}$; in 2 and 3, the height is between 4 and $8 \mathrm{~mm}$. The expectation is that for support locations close to the center, i.e., for small $x_{0}$, the positive parabola stiffness is larger than the negative parabola stiffness, but for support locations further from the center, the positive parabola stiffness increases less steeply compared to the negative parabola stiffness. The uniform leaf spring should have the highest overall stiffness, since it has the largest dimensions over its full length. The experimental setup of Figure 13 was used to measure the stiffness characteristic of the VSM. It is similar to the VSM itself, but now, the output has a handle with which it can be rotated. On the bottom, an ATI Mini40 force/torque sensor (SI-80-4 calibration) is fixed between the rotor and the fixed world, and moreover, an AMS5048A magnetic absolute angular encoder with a resolution of 14 bits is placed on top of the setup to measure the output angle. A measurement is done by placing 
the correct leaf springs, setting the correct support location and rotating the output once from its neutral position in the positive direction, back to its neutral position, in the negative direction and back to its neutral position. In this way, any non-symmetrical behavior and the possible hysteresis effect are made explicit. The two sensors are interfaced with MATLAB Simulink, where the force/torque and angle data are acquired simultaneously and processed further. A deflection/torque diagram can be obtained directly from such a measurement for certain $x_{0}$ settings, and a stiffness/support location diagram is obtained by a linear least squares approximation to this data. The slope is then the compliance, and its reciprocal is the stiffness at that $x_{0}$ setting.

Figure 12. Three different leaf spring shapes, shown in the $x-y-p l a n e$. Leaf Spring 1 is a uniform beam, and Leaf Springs 2 and 3 are beams with a negative and positive parabola cutout, respectively.

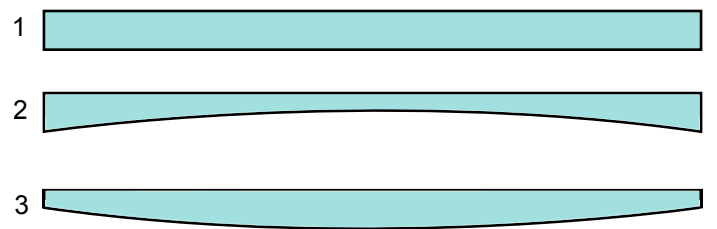

Figure 13. The experimental setup used to measure the stiffness characteristic of the variable stiffness mechanism. A magnetic sensor is placed over the device to measure the output rotation, while a force/torque sensor is placed under the device to measure the applied torque. The support pins can be placed at predefined locations.
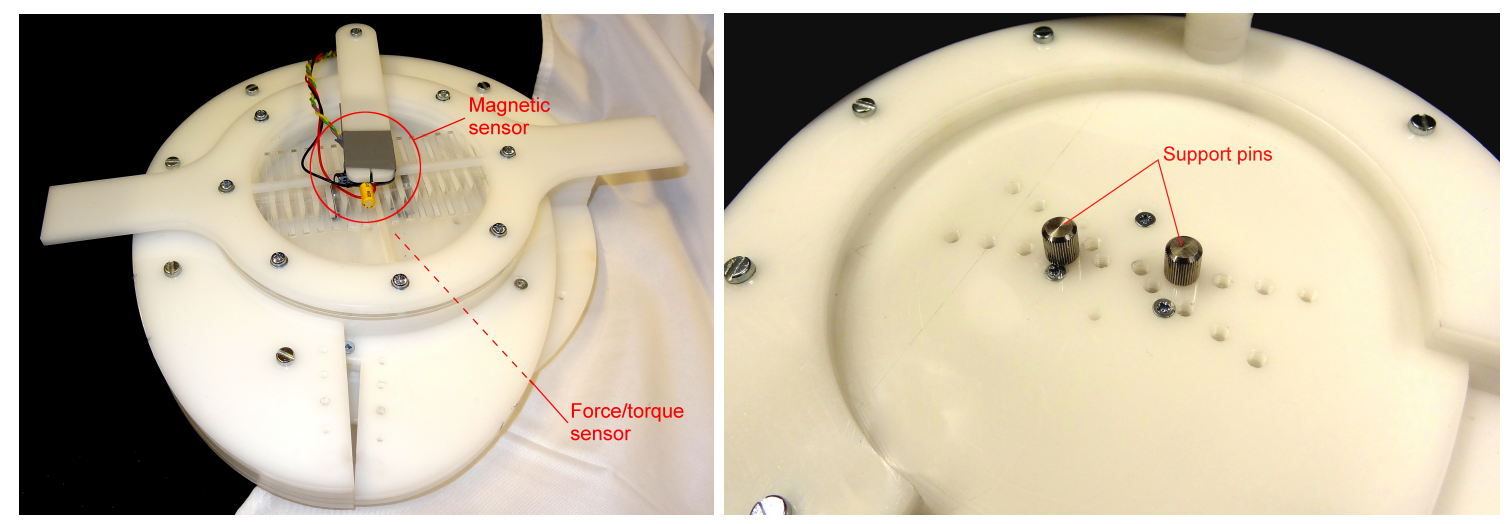

Since the model assumes only one leaf spring, also one leaf spring was first measured in the setup. Since the mechanism is designed for using a double-leaf spring, a single-leaf spring was fixed to the output and placed in between the two supports with a slight pretension, such that it is possible to measure the torque/deflection characteristic in one direction. See Figure 14 for (part of) the measurement results. Measurement data is shown for five different support location settings. The simulated torque/deflection data is shown with dashed light grey lines. A dotted line in the same color as the measurement data indicates the linear least squares approximation to the data. Figure 15 shows the support location/stiffness plots derived from Figure 14. Again, the dashed light grey line is the simulated 
stiffness, and the solid blue line is the measured stiffness. It can be seen that the experiments show the same characteristic as the simulations, and the experiments thereby validate the model in Section 3.

Figure 14. Deflection/torque measurement results using a single-leaf spring (for clarity, only half of the measurements are shown). The dashed line represents the simulated deflection/torque data, and the dotted line is the linear least squares approximation to the deflection/torque data. (a) Uniform; (b) negative parabola; (c) positive parabola.

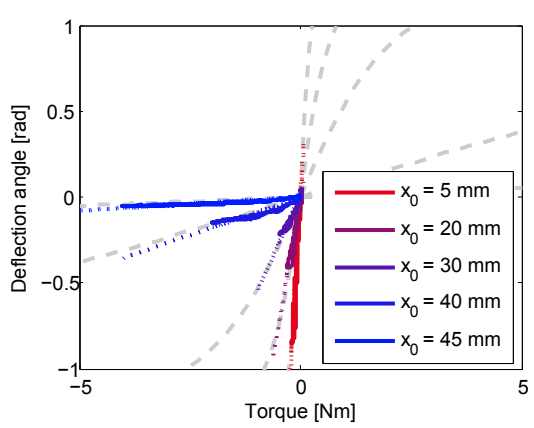

(a)

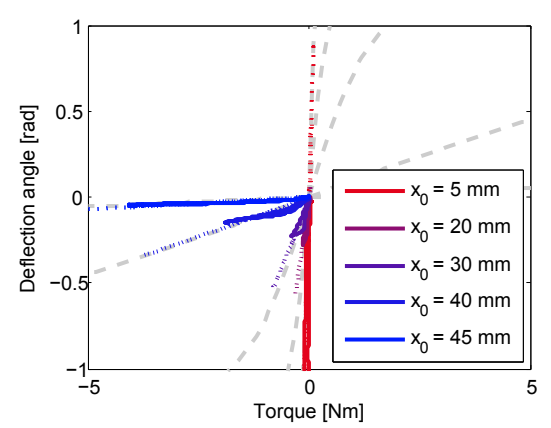

(b)

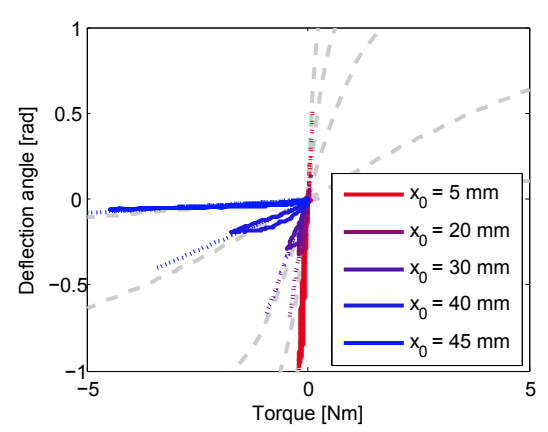

(c)

Figure 15. Stiffness/support location plots for a single-leaf spring based on a linear least squares approximation to the deflection/torque data. (a) Uniform; (b) negative parabola; (c) positive parabola.

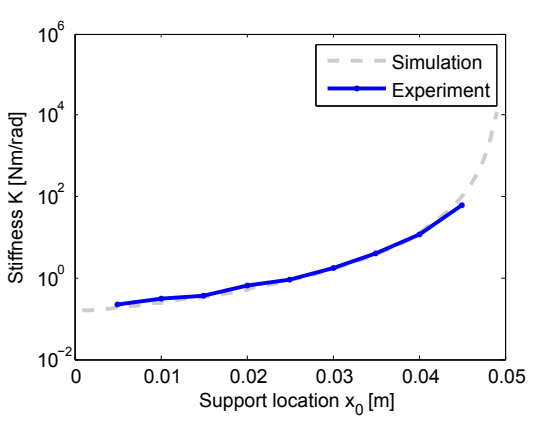

(a)

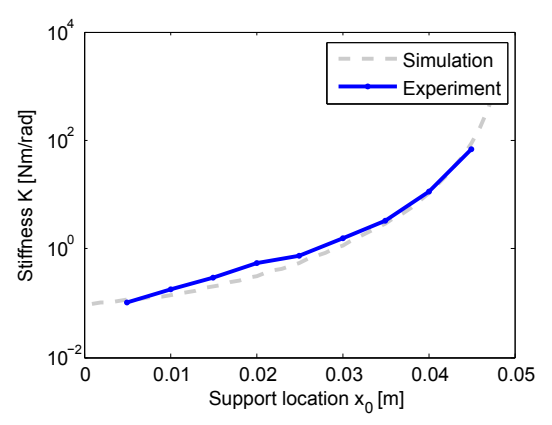

(b)

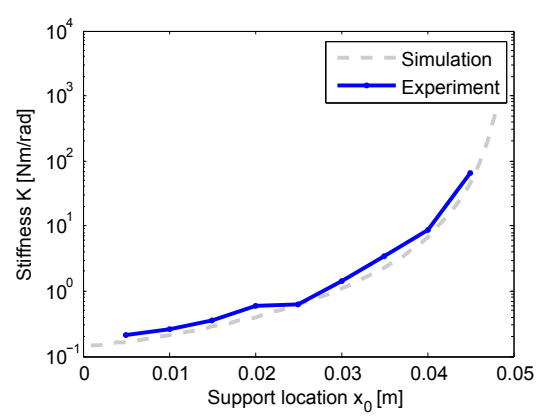

(c)

Using the same measurement procedure, the situation for the regular configuration of two leaf springs was measured, and these measurements were again used to obtain a support location/stiffness plot. Modeling the effect of a double-leaf spring constrained by clamps would likely result in a highly complex model, so this effect was empirically tested by normalizing the double-leaf spring stiffness at each support location to the stiffness obtained when using only one leaf spring, for every leaf spring shape. This results in Figure 16. These normalized stiffnesses are approximated by a linear regression for increasing support location $x_{0}$, averaged over the three leaf spring shapes, shown in dashed light grey. With this effect, the model data is adapted to incorporate the coupling to a second leaf spring. 
Figure 17 shows (part of) the torque/deflection plots using a double-leaf spring, presented in Section 2. Again, the dashed light grey lines are the simulated data, which are now adapted to incorporate the coupling of the second leaf spring, and the dotted lines in the same color as the measurement data are the linear least squares approximations. These approximations are used to calculate the stiffness using a particular leaf spring shape, which is shown in Figure 18. The dashed line is the adapted simulation data, and it is closely approximated by the experimental data.

Figure 16. Normalized stiffness derived from the double-leaf spring measurement, normalized to the single-leaf spring measurement, which shows the effect of the double-leaf spring and clamps. This effect is approximated by a linear regression for increasing support location $x_{0}$, averaged over the three leaf spring shapes.

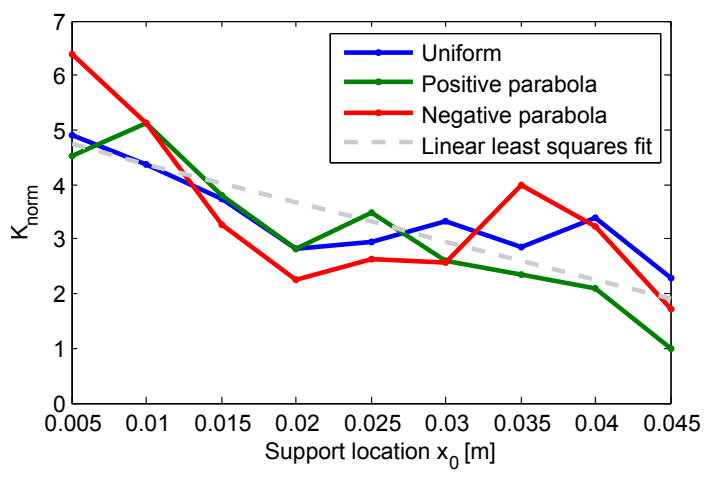

Figure 17. Deflection/torque measurement results using a double-leaf spring (for clarity, only half of the measurements are shown). The dashed line represents the simulated deflection/torque data, and the dotted line is the linear least squares approximation to the deflection/torque data. (a) Uniform; (b) negative parabola; (c) positive parabola.

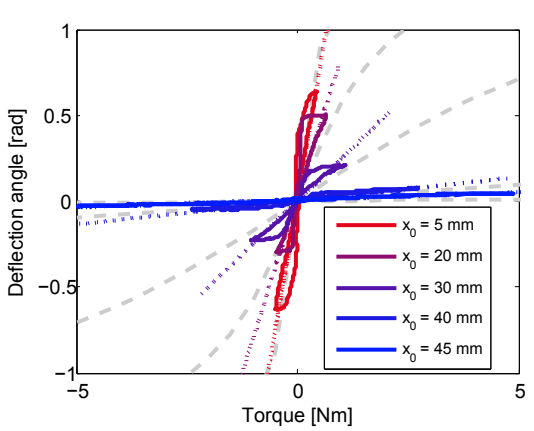

(a)

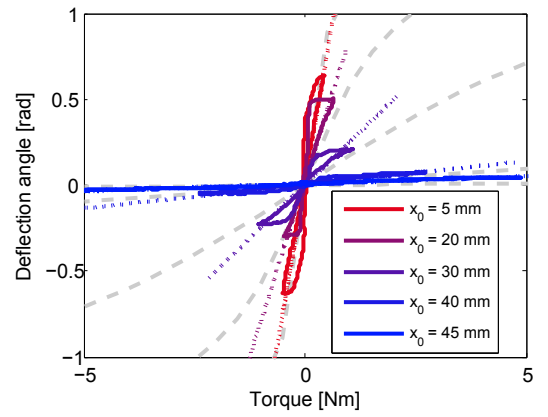

(b)

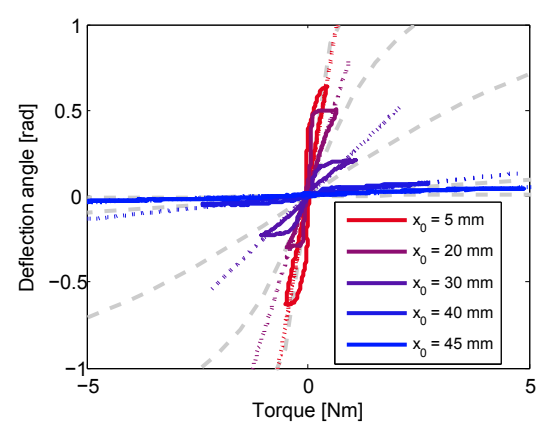

(c) 
Figure 18. Stiffness/support location plots for a double-leaf spring based on a linear least squares approximation to the deflection/torque data. The simulated line is adapted using the measured effect of the double-leaf spring and the clamps with respect to a single-leaf spring. (a) Uniform; (b) negative parabola; (c) positive parabola.

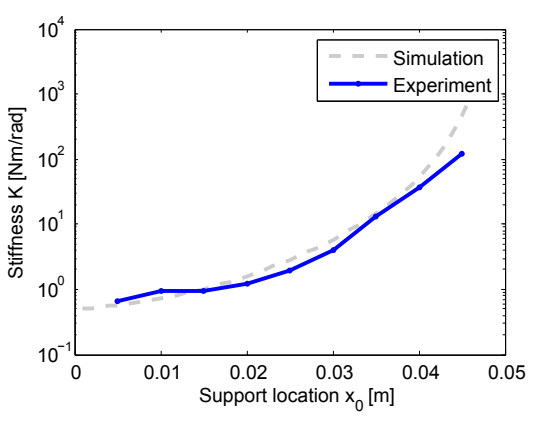

(a)

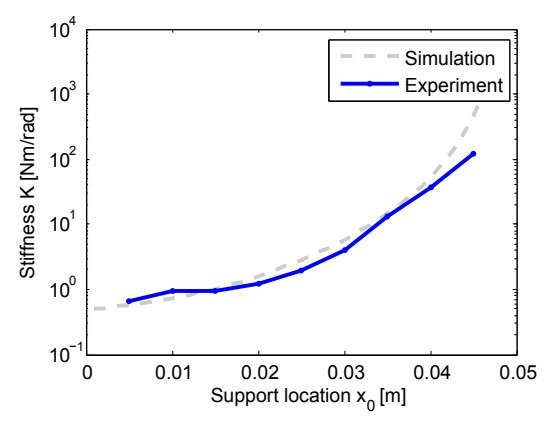

(b)

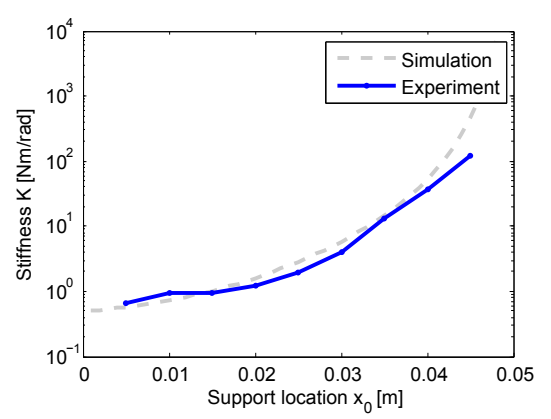

(c)

Figure 19. Stiffness plots for the three different leaf spring shapes, making the possibility of shaping the stiffness characteristic of the variable stiffness mechanism (VSM) explicit.

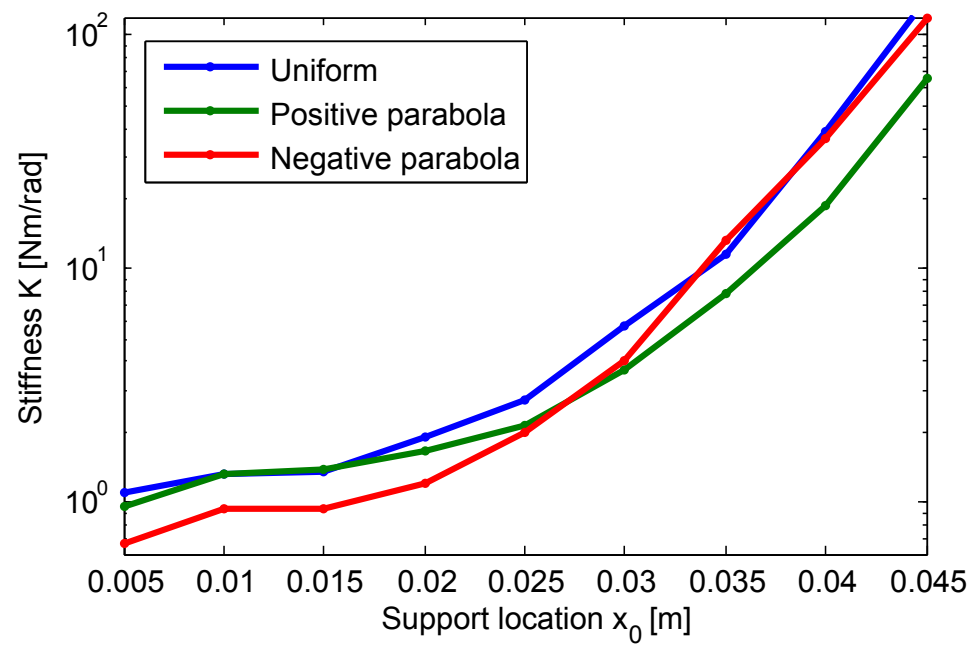

In Figures 14 and 17, hysteresis can be seen. This is due to the realization of the test setup, which has friction between the rotatable output and stator, and the stiction of the leaf spring in the groove that connects it to the output to allow the shortening of the leaf springs under deflection. Hence, this is not an intrinsic property of the mechanism. Figure 19 overlays the three stiffness plots of Figure 18 to show the ability that the stiffness characteristic of the VSM can be changed easily by shaping the leaf springs. Although for these shapes, the effect is not that profound, the stiffness shaping possibility is obviously seen when comparing the two non-uniform leaf springs: the positive parabola starts with a higher stiffness than the negative parabola, but increases less steeply, such that the negative parabola stiffness exceeds the positive parabola stiffness at $x_{0}=27 \mathrm{~mm}$. Moreover, the positive parabola stiffness for small support locations is the same as the stiffness of the uniform leaf spring, since the positive parabola has almost equal dimensions for small support locations. This is indeed according to the 
expectation. More leaf spring shapes can be explored, to achieve, for instance, a stiffness characteristic that is linear over a maximal interval.

\section{Conclusions}

In this paper, a novel variable stiffness mechanism has been presented, which is capable of an infinite stiffness range not before encountered in this class of variable stiffness realization and completely decoupled unlimited output motion with respect to the rotor for safe passive behavior. An important feature of this mechanism is the ability to easily change the stiffness characteristic by shaping the leaf springs. A Euler-Bernoulli beam model is proposed to model one of the two leaf springs that are present in the mechanism. Experiments using PETG copolyester show the validity of this model for single-leaf springs of various shapes. The stiffness measurement using the double-leaf spring is normalized to the single-leaf spring stiffness measurement, to measure the effect of coupling a leaf spring by clamps to another leaf spring. This effect is approximated and used to adapt the model data to correspond to the double-leaf spring measurements. In this way, a close approximation of the measurements to the model data is achieved. The result of shaping the leaf springs is shown and agrees with the expectation.

\section{Outlook}

A different realization of the same concept might be explored in future research, possibly one that uses only one leaf spring and one circular support with a groove, in which the leaf spring is placed, that is mounted with a bearing on the planet gear. Furthermore, a fixed support with two quarter cylinder cut-outs may be possible. Note that these different realizations do not change the properties of the mechanism, and it should be investigated whether these realizations have advantages over the presented mechanism. See Figure 20 for a schematic representation.

Figure 20. Alternative realizations of the VSM concept. One uses a pin with a groove; the other uses a fixed cylindrical pin with two quarter cylinders cut away (white parts).

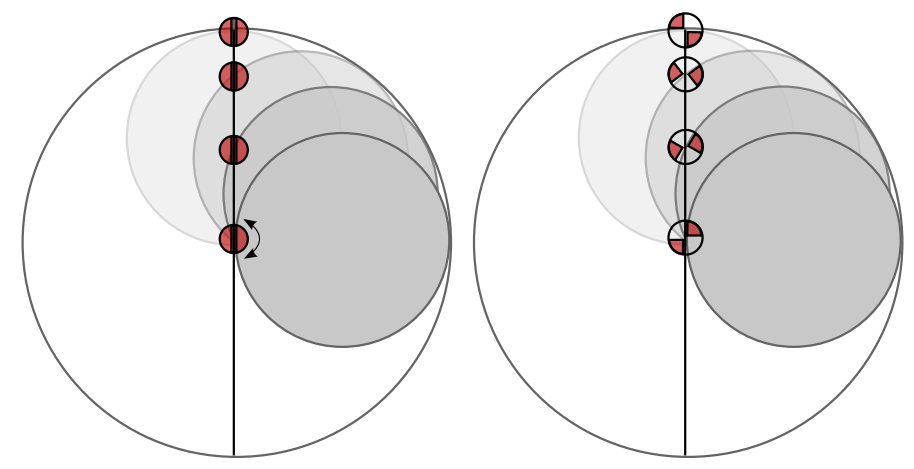

\section{Acknowledgments}

Gerben ter Riet o/g Scholten is acknowledged for his contributions in the prototype design. 


\section{Author Contributions}

The research and development of the prototype was done by Stefan Groothuis, based on the preliminary conceptual foundation by Stefano Stramigioli. Raffaella Carloni co-advised Stefan Groothuis and she was involved in the previously published conceptual designs that have led to the work presented in this manuscript.

\section{Conflicts of Interest}

The authors declare no conflict of interest.

\section{References}

1. Hogan, N. Impedance control: An approach to manipulation: Part I, part II, part III. ASME J. Dyn. Syst. Meas. Control 1985, 107, 17-24.

2. Pratt, G.A.; Williamson, M. Series Elastic Actuators. In Proceedings of the IEEE/RSJ International Conference on Intelligent Robots and Systems, Pittsburgh, PA, USA, 5-9 August 1995.

3. Vanderborght, B.; Albu-Schaeffer, A.; Bicchi, A.; Burdet, E.; Caldwell, D.; Carloni, R.; Catalano, M.; Eiberger, O.; Friedl, W.; Ganesh, G.; et al. Variable impedance actuators: A review. Robot. Auton. Syst. 2013, 61, 1601-1614.

4. Uemura, M.; Kawamura, S. Resonance-based motion control method for multi-joint robot through combining stiffness adaptation and iterative learning control. In Proceedings of the IEEE International Conference on Robotics and Automation, Kobe, Japan, 12-17 May 2009; pp. 1543-1548.

5. Groothuis, S.; Stramigioli, S.; Carloni, R. Lending a helping hand: Towards novel assistive robotic arms. IEEE Robot. Autom. Mag. 2013, 20, 20-29.

6. Vanderborght, B.; Tsagarakis, N.; Semini, C.; van Ham, R.; Caldwell, D. MACCEPA 2.0: Adjustable compliant actuator with stiffening characteristic for energy efficient hopping. In Proceedings of the IEEE International Conference on Robotics and Automation, Kobe, Japan, 12-17 May 2009.

7. Schiavi, R.; Grioli, G.; Sen, S.; Bicchi, A. VSA-II: A novel prototype of variable stiffness actuator for safe and performing robots interacting with humans. In Proceedings of the IEEE International Conference on Robotics and Automation, Pasadena, CA, USA, 19-23 May 2008.

8. Wolf, S.; Hirzinger, G. A new variable stiffness design: Matching requirements of the next robot generation. In Proceedings of the IEEE International Conference on Robotics and Automation, Pasadena, CA, USA, 19-23 May 2008.

9. Kim, B.S.; Song, J.B. Hybrid dual actuator unit: A design of a variable stiffness actuator based on an adjustable moment arm mechanism. In Proceedings of the IEEE International Conference on Robotics and Automation, Anchorage, AK, USA, 3-7 May 2010.

10. Tsagarakis, N.G.; Sardellitti, I.; Caldwell, D.G. A new variable stiffness actuator (CompAct-VSA): Design and modelling. In Proceedings of the IEEE International Conference on Intelligent Robots and Systems, San Francisco, CA, USA, 25-30 September 2011. 
11. Jafari, A.; Tsagarakis, N.; Vanderborght, B.; Caldwell, D. A novel actuator with adjustable stiffness (AwAS). In Proceedings of the IEEE/RSJ International Conference on Intelligent Robots and Systems, Taipei, Taiwan, 18-22 October 2010.

12. Jafari, A.; Tsagarakis, N.; Vanderborght, B.; Caldwell, D. AwAS-II: A new actuator with adjustable stiffness based on the novel principle of adaptable pivot point and variable lever ratio. In Proceedings of the IEEE International Conference on Robotics and Automation, Shanghai, China, 9-13 May 2011.

13. Visser, L.C.; Carloni, R.; Stramigioli, S. Energy efficient variable stiffness actuators. IEEE Trans. Robot. 2011, 27, 865-875.

14. Groothuis, S.; Rusticelli, G.; Zucchelli, A.; Stramigioli, S.; Carloni, R. The variable stiffness actuator vsaUT-II: Mechanical design, modeling and identification. IEEE/ASME Trans. Mechatron. 2013, 19, 589-597.

15. Morita, T.; Sugano, S. Development of an anthropomorphic force-controlled manipulator WAM-10. In Proceedings of the IEEE International Conference on Advances Robotics, Monterey, CA, USA, 7-9 July 1997.

16. Choi, J.; Hong, S.; Lee, W.; Kang, S.; Kim, M. A robot joint with variable stiffness using leaf springs. IEEE Trans. Robot. 2011, 27, 229-238.

17. Hollander, K.; Sugar, T.; Herring, D. Adjustable Robotics Tendon using a "Jack Spring"TM . In Proceedings of the International Conference on Rehabilitation Robotics, Chicago, IL, USA, 28 June-1 July 2005.

18. Carloni, R.; Visser, L.; Stramigioli, S. Variable stiffness actuators: A port-based power-flow analysis. IEEE Trans. Robot. 2012, 28, 1-11.

(C) 2014 by the authors; licensee MDPI, Basel, Switzerland. This article is an open access article distributed under the terms and conditions of the Creative Commons Attribution license (http://creativecommons.org/licenses/by/3.0/). 